\title{
Newer intubation techniques: a journey
}

\section{Opinion}

Tracheal intubation is the placement of a flexible plastic tube into the trachea through mouth or nose. This tube acts as a conduit to deliver oxygen and other gases from an external source like a gas cylinder, Ventilator or an Anaesthesia machine into the lungs. It is a widely used practice for providing mechanical ventilation to critically ill patients in ICU and Emergency Rooms. Intubation is also used to deliver Anaesthetic gases to unconscious patients in operation theatres and to prevent soiling of lungs by gastric secretions in comatose patients. It is also the primary step in all types of resuscitation treatments in trauma and cardiac arrest situations.

The first or tracheal intubation was reported by Sir William Macewen ${ }^{1}$ as a measure for providing ventilation in a patient of glottis oedema and for administering Chloroform anaesthesia. But during that time, visualisation of the glottis for inserting the tube into the trachea was done indirectly, by using a combination of mirrors. In 1913 Chevalier Jackson ${ }^{2}$ used direct laryngoscopy with the help of a blade that had a light source at the distal tip. This made intubation a lot easier than what was done earlier. Further modification in the design of the laryngoscope blade and the laryngoscope were done by Sir Ivan Magill ${ }^{3}$ who also designed the Magill Forceps. The laryngoscope which is used now a day was introduced by Sir Robert Macintosh ${ }^{4}$. It was a curved blade, with a distal light source and phalange to create space in the mouth. For more than half a century now, his design has remained unchallenged and his instrument has remained as the handiest and widely used device for intubation in all areas of the Operation Theatre, ICUs and emergency rooms worldwide.

But despite of the universal design, the laryngoscope failed to intubate in some patients, as the vocal cord visualisation was insufficient or sometimes even impossible. Many unexpected deaths occurred in the Operation Theatres and emergency rooms, just because the anaesthesiologists were unable to visualise the vocal cords and intubate the patients. The patients were not timely ventilated and died on the OT table itself, even before the beginning of the surgery. So, there was a search for an 'aid' which could facilitate the process of intubation. In 1949, Sir Macintosh ${ }^{5}$ published a case report describing the use of a gum elastic urinary catheter as an endotracheal tube introducer. Inspired by his report, in 1973, P Hex Venn ${ }^{6}$ developed Eschmann endotracheal tube introducer Later on, the concept of intubation stylet was introduced in 1978, by Finucane \& Kupshik who used Central Venous Catheter for the purpose. Now a day we have many types of intubation aids like Gum elastic Bougie, Frova Intubation Catheter, Flexitip stylets, Light wands etc, which have increased the manuverbility of the tube inside the oral cavity and have made difficult intubations quite possible to negotiate.

Another breakthrough in the history of intubation was the invention of Fibreoptic bronchoscope by Shigeto Ikeda ${ }^{8}$. Though it was invented, to visualise the pathologies of tracheobronchial tree, but because its manuveribility and due to its thin size of 5-6 $\mathrm{mm}$, it became an essential component of every Anaesthesia Department's 'Difficult Airway Cart'. With the help of fiber optic bronchoscope, virtually every case of difficult intubation, like patients of facial burns, syndromic babies who had their airway anatomy distorted, was now possible and within Anaesthetist reach. But despite of these
Volume 6 Issue 3 - 2018

\author{
Abhinav Gupta, Shalu Singh, Harikishan \\ Mahajan \\ Machine Learning Department, Carnegie Mellon University, USA
}

Correspondence: Abhinav Gupta, Machine Learning

Department, Carnegie Mellon University, USA

Emaildrabhinav.isic@gmail.com

Received: March 26, 2018 | Published: May 08, 2018

advantages in intubation, the bronchoscope had the drawback of high cost and requirement of more time for assembly and cleaning, which made it difficult to use in Emergencies and unexpected difficult intubations. Its camera if it was soiled with blood, made it very difficult to visualise the vocal cords and intubate the patients. The patient had to be awake and cooperative for the procedure to which caused discomfort. Nevertheless, Fiberoptic bronchoscope has been a very useful device for all cases of planned difficult airway management cases in the Operation Theatre and ICU.

Another new development in the history of intubation was the incorporation of cameras, LCD screens and digital technology in the laryngoscopes. These new devices are based on indirect laryngoscopy instead of Direct Laryngoscopy of the Macintosh Blade. A live video of the glottisis available on the screen, which can be seen not only by the anaesthetist who is performing the procedure but other persons in the OT also. These videos can be recorded and snapshots can be taken for teaching purposes. Many of these Video laryngoscopes have channels to carry the tube to the glottic opening and special shapes which have reduced the difficulty in difficult intubation cases. King Vision Videolaryngoscope ${ }^{9}$ which is marketed by Ambu has a channel to carry the tube and is useful, where larynx is anteriorly placed and neck movement is restricted. Its blades are disposable providing better hygiene in infected patients. It has an added advantage of being low cost and handy. C-Mac D blade laryngoscope (by Storc) ${ }^{9}$ requires less mouth opening for insertion of the blade and has better success rate in anterior larynx. It has internal rechargeable battery and has buttons for taking screenshots. In Vividtrach, ${ }^{9}$ images are displayed on a connected laptop, which can be seen by other people also. And according to manufacturer there is no need to tilt the head during intubation. But despite these advantages, these video laryngoscopes have a certain learning curve and require some amount of practice before one achieves $100 \%$ success with them. They also have issues in cases where there is blood or too many secretions in the oral cavity because these soils the camera lens and obscure the vision, making intubation difficult. The cost of these newer devices is in between 1 to 5lacs. King Vision is a low-cost alternative and is available at a price below 1lac. But its blades are disposable which increase the usage prices. Nevertheless, Video laryngoscopes have proved to be very handy and useful devices. They can be assembled within seconds and intubation can be achieved within 15 to 30 seconds similar to that of Macintosh Laryngoscope. In cases where patient's oral anatomy is normal, these devices are now proving to be even superior to the Fiberoptic bronchoscope. 


\section{Acknowledgements}

None.

\section{Conflict of Interest}

Author declares that there is no conflict of interest.

\section{References}

1. Brandt L. The history of endotracheal anesthesia, with special regard to the development of the endotracheal tube. Anaesthesist. 1986;35(9):523530 .

2. Ezri T1, Evron S, Hadad H, et al. Tracheostomy and endotracheal intubation: a short history. Harefuah. 2005;144(12):891-908.

3. W Magill. TECHNIQUE IN ENDOTRACHEAL ANAESTHESIA. $\mathrm{Br}$ Med J. 1930;2(3645):817-819.
4. MacIntosh R. "A NEW LARYNGOSCOPE". The Lancet. 1943;241(6233):205.

5. “REPORTS OF SOCIETIES”. BMJ. 1949;1(4591):26.

6. Venn PH. The gum elastic bougie. Anaesthesia.1973;48(3):274-275.

7. Finucane BT, Kupshik HL. A flexible stilette for replacing damaged tracheal tubes. Canadian Anaesthetists 'Society Journal. 1978;25(2):153154.

8. Miyazawa T. Historyof the flexible bronchoscope. In: Bolliger CT, Mathur PN, editors. Progress in respiration research. Vol. 30: Interventional bronchoscopy. Basel, Switzerland: Karger; 2000. p. 16-21.

9. Cierniak M, Timler D, Wieczorek A, et al.The comparison of the technical parameters in endotrachealintubation devices: the Cmac, the Vividtrac, the McGrath Macand the Kingvision. J ClinMonitComput. (2016);30(4):379387. 